\title{
Acteurs tiers et stratégies collectives au sein des filières. Le cas du liège en Corse
}

Third party and collective strategies within chains: the cork case in Corsica

\section{Laetitia Dari et Gilles Paché}

\section{(2) OpenEdition}

\section{Journals}

\section{Édition électronique}

URL : http://journals.openedition.org/economierurale/4750

DOI : $10.4000 /$ economierurale. 4750

ISSN : 2105-2581

\section{Éditeur}

Société Française d'Économie Rurale (SFER)

\section{Édition imprimée}

Date de publication : 15 décembre 2015

Pagination : 101-123

ISSN : 0013-0559

\section{Référence électronique}

Laetitia Dari et Gilles Paché, «Acteurs tiers et stratégies collectives au sein des filières. Le cas du liège en Corse », Économie rurale [En ligne], 349-350 | septembre-novembre 2015, mis en ligne le 01 décembre 2017, consulté le 30 avril 2019. URL : http://journals.openedition.org/economierurale/4750 ; DOI : 10.4000/economierurale.4750 


\title{
Acteurs tiers et stratégies collectives au sein des filières Le cas du liège en Corse
}

\author{
Laetitia DARI, Gilles PACHÉ • Centre de Recherche sur le Transport et la Logistique (CRET-LOG), \\ Aix-Marseille Université \\ laetitia.dari@univ-amu.fr; gilles.pache@univ-amu.fr
}

Les relations inter-organisationnelles ne sont pas uniquement constituées de tension et de concurrence. Lorsqu'une instabilité sectorielle existe, les entreprises peuvent chercher à se regrouper autour d'acteurs intermédiaires, représentatifs du secteur dans lequel elles évoluent. Ces acteurs tiers vont permettre de redynamiser une filière, à condition que leur légitimité soit reconnue. L'article s'intéresse au cas de la filière du liège en France. II en ressort que si, sur le Continent, des organismes collectifs œuvrent de manière efficace dans la dynamisation de la filière, il n'en va pas de même en Corse, où les stratégies collectives ont pour l'instant échoué. Les raisons de l'échec sont mises en lumière, puis discutées à partir d'une grille d'analyse des stratégies collectives.

MOTS-CLÉS : acteur tiers, coopérative, filière, liège, stratégies collectives

\section{Third party and collective strategies within chains: the cork case in Corsica}

Inter-organizational relations are not only constituted by tension and by competition. When a sectorial instability exists, companies can cooperate around intermediate, which are representative actors of the sector. These third party are going to re-revitalize a sector, provided that their legitimacy is recognized. The article is based on the case of cork industry in France. If on the Continent, collective organizations work in an effective way to revitalize the sector, it is not also go away in Corsica, where collective strategies are a failure. The reasons of the failure are highlighted, then discussed from an analysis of collective strategies. (JEL: L10, M10, Q23).

KEYWORDS: third party, cooperative, chain, cork, collective strategies

$\mathbf{L}^{2}$ complexité et l'instabilité de l'environnement dans lequel évoluent aujourd'hui les entreprises ne sont pas sans impact sur la nature des relations entre organisations et sur la gouvernance de ces relations. Co-construire des systèmes d'offre et, plus largement, poursuivre des stratégies collectives de création de valeur s'avère être particulièrement difficile lorsque la brutalité des turbulences fait s'interroger sur la capacité de l'un ou plusieurs des partenaires à poursuivre l'effort qui leur est réclamé. Ces partenaires peuvent être également tentés de rejoindre un autre projet plus rémunérateur ou présentant moins de risques. Dans un contexte marqué par l'extrême plasticité de relations d'affaires, qui peuvent se faire et se défaire au gré de multiples opportunités, la présence d'acteurs intermédiaires jouant le rôle de régulateur et de stabilisateur des échanges retient de plus en plus significativement l'attention. Des recherches menées sur le sujet soulignent ainsi la place centrale que tiennent ces acteurs dans le développement et la structuration à long terme d'un secteur d'activité (Astley, Fombrun, 1983 ; Geindre, 2005 ; Yami, Le Roy, 2006 ; Loubaresse, Pestre, 2014).

D'une manière générale, il est question d'acteurs d'interface, ou encore d'acteurs tiers, dont la mission est d'assurer une fonction de médiation permettant de gouverner plus efficacement les stratégies 
collectives, tout particulièrement dans le cadre de règles du jeu solidairement acceptées (Orléan, 1994 ; Geindre, 2005 ; Gadille, 2008 ; Zaoual, 2014). Cette fonction peut être exercée simultanément ou successivement par des organismes collectifs, des syndicats professionnels, des fédérations ou encore des associations qui, compte tenu de leurs caractéristiques, trouvent une légitimité à leur existence dans la dynamisation d'un écosystème d'affaire de type filière, l'écosystème étant entendu ici comme une communauté économique fondée sur l'interaction entre des organisations qui produisent des biens et des services en apportant de la valeur aux clients, d'ailleurs eux-mêmes partie prenante dudit écosystème (Moore, 1993). Par légitimité, il faut comprendre, à la suite de Suchman (1995), la capacité d'un acteur à adopter le comportement approprié et souhaitable vis-à-vis d'un système social de normes. Il en résulte de nombreuses interrogations sur les sources d'une telle légitimité et sur les ressorts pouvant conduire un acteur tiers à jouer (ou non) un rôle clé dans la relance possible d'une filière confrontée à des problèmes de compétitivité. L'objectif de l'article est justement de s'intéresser aux rôles et missions de l'acteur tiers en tant qu'intermédiaire et d'évaluer la dynamique dont il peut être porteur dans la réussite d'une stratégie collective.

La recherche s'appuie sur une étude de cas : celui de la filière du liège en France. Laissée à l'abandon à partir des années 1950, concurrencée par des produits de substitution (Yessad, 2000 ; Botey, 2012), il subsiste pourtant au sein de la filière des acteurs économiques et politiques qui œuvrent activement afin de lui donner une nouvelle vie. La tendance à la consommation de produits naturels et écologiques, soulignée par Moisander (2007), ainsi que les nombreux atouts intrinsèques du liège, constituent il est vrai autant de raisons objectives pour envisager des actions structurantes permettant la relance de la filière. Le liège possède de nombreuses qualités qui font de lui un produit naturel unique : imperméable, imputrescible, autoextinguible, isolant thermique et phonique, et léger. S'agissant d'une matière première au potentiel multiple, de nombreux débouchés peuvent être envisagés, par exemple en termes de bouchon, d'isolant, de revêtement, de vêtement, d'élément de décoration. Il dispose également de nombreux avantages économiques en termes de coût de production.

L'article s'intéresse tout particulièrement au cas de la Corse suite à l'adoption, en février 2013, d'une motion par l'Assemblée de Corse relative au développement de la subériculture sur l'île. Le chêne-liège constitue en effet un patrimoine naturel et culturel propre dont la Corse est dotée, constituant à elle seule 30000 hectares, pour seulement 15000 hectares exploités. La subériculture insulaire, qui était l'une des plus importantes avec celle du Var jusque dans les années 1950, tente désormais de se maintenir avec quelques propriétaires forestiers et négociants. L'activité industrielle a, quant à elle, totalement disparu et s'effectue en Sardaigne ou au Portugal. Il est également important de noter les enjeux environnementaux liés à la relance de cette filière dans la mesure où l'abandon de la subériculture a provoqué un fort embroussaillement des parcelles, augmentant de ce fait les risques d'incendie sur l'île. Enfin, le manque d'information sur les aides accessibles aux propriétaires de suberaies (desserte de leurs parcelles par des pistes forestières, élagage, récolte de liège dégradé, etc.) ne permet pas d'enclencher une réelle dynamique de reprise de l'activité.

L'analyse de la filière du liège met en évidence l'existence d'organismes collectifs dont les actions ont pour objet de relancer progressivement l'activité liège. Or l'étude du cas singulier de la Corse 
tend à indiquer que, malgré des potentialités évidentes sur le territoire insulaire, les organismes collectifs ont finalement peu d'impact positif. Comment l'expliquer ? Une analyse s'appuyant sur les apports théoriques du concept d'acteur tiers met en lumière un certain nombre de défaillances. La question est alors de savoir si des relations inter-organisationnelles ont toujours besoin d'être coordonnées par des acteurs intermédiaires pour réussir, et si oui, comment la forme organisationnelle de ces acteurs (coopératives, associations, etc.) joue sur leur efficacité. L'objectif est d'effectuer un retour d'expérience sur les bonnes et mauvaises pratiques collectives observées, à partir d'une étude de terrain approfondie qui s'est déroulée sur plusieurs mois. L'enjeu managérial est de mettre en lumière un possible effet d'apprentissage offrant aux entreprises de la filière des opportunités de développement. Pour cela, nous exposerons dans un premier temps les aspects théoriques liés à la notion d'acteur tiers. Dans un deuxième temps, nous préciserons la méthodologie de recherche retenue avant de présenter, dans un troisième temps, les principaux résultats. Dans un quatrième et dernier temps, nous procéderons à une discussion fondée sur un rebouclage théorique ${ }^{1}$.

\section{L'acteur tiers dans le management des relations inter-organisationnelles}

Étudier le concept d'acteur tiers nécessite de se pencher préalablement sur la question des institutions et de leur rôle dans la gouvernance des relations d'échange entre organisations. Le rôle des institutions est

1. Les auteurs remercient chaleureusement deux évaluateurs anonymes d'Économie rurale et Pierre Labarthe pour la qualité de leurs commentaires et suggestions, qui ont permis d'améliorer de manière significative les versions antérieures de l'article. Tous nos remerciements également au GIP Corse Compétences pour le financement de la présente recherche. analysé différemment selon les courants institutionnalistes auxquels on se réfère (Théret, 2000). Il peut renvoyer alternativement au cadre dans lequel s'inscrit la coordination entre acteurs, dans la théorie des jeux non coopératifs (Walliser, 1989), à des structures formelles de coordination, dans le courant néo-institutionnaliste (Powell, DiMaggio, 2012), ou encore à des règles, conventions ou normes de comportement intervenant avant et pendant la coordination, dans l'économie des conventions (Bessy, Favereau, 2003), voire à l'expression de rapports de force entre acteurs (Boyer, 2003). Pour notre part, nous retiendrons une conceptualisation néo-institutionnaliste de l'institution en référence à la convention nouée entre deux acteurs permettant d'équilibrer leurs relations (North, 1990 ; Aoki, 2001), notamment par la mise en place d'un environnement structuré par des « règles du jeu » (Davis, North, 1971 ; Ménard, 2003). Ces règles du jeu permettent de réguler les rapports entre entreprises et de définir la manière dont les transactions doivent s'effectuer entre elles. Sur un plan plus managérial, le rôle d'une institution sera de coordonner des entreprises impliquées dans un projet commun et d'assurer une médiation efficace entre elles. Trois points clés sont ainsi à mettre en avant (Ménard, 2003) :

- une institution se caractérise par des règles et des normes stables et durables, en référence à un horizon temporel éloigné ;

- une institution est neutre dans la mesure où les règles retenues sont abstraites et impersonnelles (Hurwicz, 1987) ;

- une institution régule les relations par les règles et les normes qu'elle définit, mais limite également la capacité de chaque entreprise à prendre des décisions.

Dès lors que se mettent en place une aire relationnelle (le jeu) et des règles lui étant affectées, les institutions exercent un pouvoir sur les acteurs et les entreprises qui les utilisent. Cette gouvernance, 
formée par une institution et fondée sur le partage de croyances et de représentations qui la légitiment, permet d'envisager une coopération entre entreprises. Les institutions deviennent ainsi un élément central de l'action collective en coordonnant les entreprises dans le respect de leurs individualités. Elles se présentent en effet comme un élément permettant la médiation entre deux parties (Orléan, 1994). Pour certains auteurs, ces institutions sont garanties par des « acteurs tiers », qui agissent « comme une garantie pour celui qui accorde sa confiance » (Geindre, 2005, pp. 77-78). Ils assurent que l'autre partie va agir favorablement et, ainsi, ne pas chercher à tirer avantage de la situation de vulnérabilité de celui qui accorde sa confiance. Cette situation suppose toutefois que les parties aient pu expérimenter la capacité du tiers à garantir le risque encouru, c'est-à-dire qu'elles ont elles-mêmes confiance dans l'action du tiers. L'acteur tiers peut dès lors favoriser l'expression et la consolidation d'apprentissages collectifs interentreprises (Gadille, 2008).

Comme le note Fourcade (2006a), l'acteur tiers est un «mentor » qui gère et coordonne la filière. Son rôle et sa position le conduisent à être garant de sa cohérence et de son efficacité. Fourcade (2006b) souligne combien l'instabilité au sein d'une filière est souvent une raison qui pousse les entreprises à coopérer. Astley et Fombrun (1983) ont ainsi montré que l'instabilité sectorielle permettait de faire émerger de nouvelles relations entre entreprises. La typologie qu'ils proposent au sujet de ces relations met notamment en évidence l'importance des stratégies collectives agglomérées, coordonnées par une structure de coordination au sens de Granata (2010), pouvant prendre la forme d'un acteur tiers, qu'il s'agisse d'un syndicat, d'une association ou encore d'une fédération. La dynamique collective instaurée par ce dernier permet ainsi de réduire l'incertitude émanant des décisions individuelles dans la mesure où chaque entreprise œuvre dans la même direction. L'acteur tiers centralise la gouvernance et assure un pilotage cohérent du tout (Yami, Le Roy, 2006 ; Le Roy, 2008) ; il constitue également un élément facilitateur d'adoption de l'innovation technique et/ou managériale par le collectif d'entreprises (Mizuno, 2014).

Malgré leur diversité institutionnelle et celle de leur aire d'influence, nationale ou régionale, les acteurs qui fédèrent un ensemble d'entreprises impliquées dans le fonctionnement d'une filière ont une mission commune : aider financièrement, promouvoir et communiquer sur la filière. Interlocuteurs essentiels en matière de promotion, de commercialisation et d'aide à l'innovation, ces acteurs tiers agissent pour le compte d'un « collectif». Gundolf et al. (2006) soulignent que les entreprises relèvent ici d'un écosystème puissant fondé sur un « encastrement institutionnel ». Autrement dit, les entreprises d'une filière se réfèrent à des structures externes qui leur permettent de bénéficier de ressources (informations, clients, lobbying, innovations, etc.) sans lesquelles leur pérennité ne pourrait être garantie, et leur position concurrentielle menacée. Les acteurs tiers occupent une place stratégique en constituant le « ciment » de la stratégie collective : ils permettent de donner une vision homogène de la filière et limitent l'instabilité à laquelle sont confrontées les entreprises. Ils jouent « un rôle "d'ombrelle", de tutelle, pour apporter une cohérence à l'action collective » (Gundolf et al., 2006, p. 151). La notion de confiance envers l'acteur tiers est donc capitale (Orléan, 1994), ceci afin de rassembler le plus grand nombre possible d'entreprises et de revendiquer une forte légitimité dans l'application des règles inhérentes au fonctionnement de la filière. Pour leur part, Greenwood et al. (2002) reconnaissent aux acteurs tiers une importance majeure dans 
la gouvernance des écosystèmes pour trois raisons essentielles :

- ils représentent des « aires relationnelles » dans lesquelles les entreprises interagissent, leur neutralité permettant de créer un consensus qui facilite les relations entre elles ;

- ils sont également garants de l'image de la filière et de ses entreprises, en communiquant auprès de multiples parties prenantes ;

- ils exercent un rôle de sanction afin d'assurer la stabilité de la filière et de garantir le suivi des règles collectivement définies.

En conclusion, l'acteur tiers se positionne au centre de gravité d'un écosystème dont la réussite dépend d'une capacité à penser collectif, indépendamment des individualismes et des égoïsmes stratégiques (Astley, Fombrun, 1983 ; Yami, Le Roy, 2006 ; Granata, 2010 ; Loubaresse, Pestre, 2014 ; Granata, Le Roy, 2014). L'un des exemples les plus illustratifs est donné par des logiques de dynamisation d'un territoire grâce à une action collective concertée autour d'un puissant projet fédérateur (Zaoual, 2014). Or, si de nombreuses recherches ont mis en lumière des succès à ce niveau, dans des filières variées, notamment dans l'agroalimentaire (Fourcade et al., 2010), il s'avère intéressant d'étudier un cas d'échec, alors même que la présence d'une unité territoriale pourrait être gage de puissantes solidarités collectives. Une telle étude de cas contribue à une meilleure compréhension des facteurs de réussite interne (type d'organisation) et externes (contexte politique, asymétries entre entreprises, etc.) des acteurs tiers. Notre investigation prend appui sur la filière du liège en France. Certaines initiatives locales, notamment en Corse, y ont été conduites pour la dynamiser, en tentant d'y développer des stratégies collectives et coordonnées (Dari, 2013, 2014). Les résultats obtenus sont mitigés ; ils justifient une investigation approfondie.

\section{Méthodologie de la recherche}

L'objectif de la recherche est d'évaluer en quoi la relance de la filière du liège en France peut ou non bénéficier de la mise en place d'acteurs tiers, agissant comme « ciment » des relations inter-organisationnelles au sein de l'écosystème. L'encadré 1 fournit quelques indications rapides sur l'industrie du liège. L'analyse des quatre régions françaises où le liège est présent tend à souligner l'importance différenciée de cet intermédiaire. Ainsi, en Aquitaine, dans les Pyrénées-Orientales et dans le Var, la présence de structures collectives de type acteur tiers participe au maintien d'une activité et agit également comme force de proposition pour la création de projets de développement. À l'inverse, le cas de la Corse met en évidence l'absence préjudiciable d'un intermédiaire évitant la diffusion de forces centrifuges. La justification d'un usage de l'étude de cas se fonde sur la complexité organisationnelle du problème à examiner, mais également sur sa dimension évolutive (Ring, Van de Ven, 1994). Selon Ring et Van de Ven (1992), l'étude de cas met ainsi en lumière plusieurs étapes distinctes : l'identification d'événements et l'interprétation des données recueillies ; l'analyse des relations de cause à effet ; la construction du cheminement logique dans une suite d'événements. Il s'agit, le plus souvent, d'une démarche de nature exploratoire devant, au final, répondre à la question de recherche en termes de comment et de pourquoi (Yin, 2013). C'est le cas ici puisque nous souhaitons comprendre le comment et le pourquoi de distorsions dans la manière de conduire des stratégies collectives au sein de la filière liège en France et, par un retour d'expérience sur le cas corse, en tirer des éléments utiles d'apprentissage. 


\section{Encadré 1. Quelques éléments sur la production du liège}

Le chêne-liège se trouve essentiellement dans les pays du pourtour méditerranéen : Portugal, Espagne, Maroc, Algérie, Tunisie, Italie et France. En France, quatre zones géographiques sont concernées : une zone atlantique (Aquitaine), et une zone méditerranéenne (Pyrénées-Orientales, Var et Corse) (Dugelay, 1952). Ressource rare, la plupart des suberaies sont désormais sous-exploitées (Groené, 1983 ; Piazzetta, 2005). Il existe 2,2 millions d'hectares de suberaies (plantations de chêne-liège) dans le monde, pour 360000 tonnes de liège produites par an (Apcor, 2006). La France représente $3 \%$ de la surface des suberaies mondiales et $1 \%$ de la production de liège (Yessad, 2000). Soit une surface de 65000 hectares et une production de liège femelle de 5000 à 7000 tonnes par an (Piazzetta, 2005). Il existe une majorité de suberaies privées (Sabate, 2000). Le Portugal, l'Espagne et le Maroc produisent $97 \%$ du liège, la France arrivant en septième position (Piazzetta, 2009).

Selon Normandin (1980), il faut attendre 25 ans avant que le chêne-liège puisse produire du liège $(70 \mathrm{~cm}$ à $1,30 \mathrm{~m}$ de circonférence). Lors de la première récolte (démasclage) est prélevé ce que l'on appelle le liège mâle, utilisé dans la fabrication d'isolants. Il faut ensuite attendre entre 9 et 12 ans pour récolter de nouveau du liège (levée de liège) ${ }^{2}$. Sur ces autres levées, le liège dit femelle, hautement qualitatif, pourra servir de liège bouchonnable. Pour des raisons de rareté de la main-d'œuvre, de non-rentabilité immédiate du produit, et d'abandon progressif des activités forestières, la plupart des surfaces sont désormais sous-exploitées (Groené, 1983 ;
Piazzetta, 2005). Ajoutons qu'il s'agit d'une ressource rare dans la mesure où il pousse seulement en Méditerranée ; les pays de cette zone disposent d'une réelle rente de situation que d'hypothétiques nouveaux entrants venus d'autres contrées ne peuvent leur contester. En bref, nous sommes dans une logique de spécificité de site, pour reprendre Williamson (1985), liée à la localisation contrainte de l'actif.

$\mathrm{Si}$, à la fin du $\mathrm{XIX}^{\mathrm{e}}$ siècle et au début du $\mathrm{XX}^{\mathrm{e}}$ siècle, les activités économiques autour du liège étaient florissantes, de nombreux éléments sont venus perturber le marché français : déclin des activités forestières et abandon des forêts, hausse du coût de la main-d'œuvre par rapport au Portugal, perte de savoir-faire, etc. Aujourd'hui, l'industrie du liège en France n'existe quasiment plus. La majorité des entreprises sont des négociants qui ne transforment pas la matière première mais la font transiter vers le Portugal, l'Espagne ou l'Italie. Le liège revient ensuite sous la forme de produits finis qui seront commercialisés par ces mêmes entreprises. Le Portugal est aujourd'hui le leader dans ce secteur, tous produits confondus (bouchons, isolants, design, etc.). Cette position est notamment due à une activité liège qui a su se perpétuer sur le territoire. Les entreprises portugaises ont également appris à se structurer autour d'organismes collectifs garants du développement de la filière, notamment Retecork (réseau européen assurant le développement des localités possédant du liège, avec un lobbying au niveau européen) et Apcor (association ayant pour vocation de représenter l'industrie du liège portugais).
L'unité d'analyse principale retenue est la filière du liège. Elle est complétée par deux autres unités d'analyse sous-jacentes que sont les relations inter-organisationnelles et les réseaux d'affaires. Afin de collecter les informations indispensables

2. La levée de liège correspond à la récolte du liège femelle alors que le démasclage (préparation) correspond à la récolte du liège mâle. pour la conduite de notre recherche, nous nous sommes basés sur des données à la fois secondaires et primaires. Les données secondaires sont constituées de sources d'information externes : rapports d'étude menés par les organismes interrogés, sites Internet, presse professionnelle, articles universitaires. Les données primaires sont constituées d'entretiens semi-directifs menés à partir d'un guide d'entretien portant 
sur l'état des lieux de la filière, les acteurs la composant, le rôle et les missions de la structure interrogée, les résultats obtenus et les difficultés rencontrées, les relations inter-structures, et les freins et leviers à l'intégration d'un collectif. Leur collecte a été interrompue lorsque nous avons constaté que les nouveaux entretiens effectués n'apportaient plus rien de nouveau, ce qui correspond au «point de saturation » de Glaser et Strauss (1967), ou encore au « principe d'achèvement » (completeness) de Rubin et Rubin (1995). Les entretiens, d'une durée moyenne de $1 \mathrm{~h} 10$, se sont majoritairement déroulés en face à face, ou par téléphone pour quelques-uns d'entre eux. Ils ont été enregistrés, puis retranscrits rapidement afin de préserver la qualité de l'information (Romelaer, 2005). Les notes prises, ainsi que nos impressions, ont également été saisies informatiquement à l'issue des entretiens. Une première lecture a été effectuée afin de prendre connaissance de l'ensemble des données. Une seconde lecture a ensuite été faite afin de mettre en relief les données par rapport aux propositions de départ. Le corpus final s'appuie sur 36 entretiens, soit environ 40 heures d'enregistrement entièrement retranscrites (voir le tableau 1).

Notre recherche, de nature qualitative, se réfère à la théorie enracinée au sens où il est question de procéder à une abstraction progressive à partir d'éléments concrets, en cernant les caractéristiques essentielles du phénomène étudié (Strauss, Corbin, 1990). L'objectif n'est pas de produire une description détaillée de différentes situations de gestion relatives à la filière $\mathrm{du}$ liège, comme pourrait y conduire une démarche de type ethnographique, mais plutôt de « découvrir » une grille de lecture conceptuelle pouvant être transférée à d'autres configurations, dans le cadre d'une théorie dite de "moyenne portée » (Guillemette, 2006). À la suite de Glaser et Strauss (1967), l'option retenue est celle de l'induction analytique, qui consiste à discuter la pertinence d'hypothèses préalablement formulées dans la littérature par confrontation avec des « cas négatifs » issus du terrain. Il s'agit de tester ici l'hypothèse selon laquelle les acteurs tiers seraient efficaces pour redynamiser des filières dans un contexte de concurrence accrue ; le « cas négatif » correspondant à la situation en Corse. Cette étude de cas permet aussi de mieux comprendre en quoi la forme organisationnelle de l'acteur tiers (ici une coopérative) joue sur son efficacité. L'analyse de contenu utilisée comporte différentes phases : la pré-analyse, l'exploitation du matériel et le traitement des résultats et leur interprétation (Bardin, 2013). La pré-analyse a pour objectif d'organiser les données de départ. Une lecture flottante des documents et entretiens met en évidence des indices qui permettent d'élaborer des thèmes et d'effectuer le codage. Certaines règles sont néanmoins à prendre en compte : l'exhaustivité (il faut tenir compte de tous les éléments), la représentativité (l'échantillon doit être représentatif d'un ensemble), l'homogénéité (les documents utilisés et les entretiens effectués doivent être identiques pour ensuite pouvoir généraliser les résultats de la recherche), et la pertinence (les données doivent correspondre aux objectifs de la recherche). Le codage a été effectué suite aux premiers entretiens. Il a ainsi permis de préparer l'analyse et d'orienter le recueil des données pour les entretiens qui ont suivi. Une fois ces étapes réalisées, le matériau a été exploité à l'aide du logiciel NVivo.

\section{Filière du liège en France Le rôle contrasté des acteurs tiers}

Un nombre important de recherches a pu démontrer que l'instabilité d'un secteur ou d'une filière peut être « régulée » par la mise en place de stratégies collectives, au sens d'Astley et Fombrun (1983). 
Acteurs tiers et stratégies collectives au sein des filières

Tableau 1. Acteurs interrogés lors de la recherche

\begin{tabular}{|c|c|c|}
\hline $\begin{array}{l}\text { Catégories et } \\
\text { organisations }\end{array}$ & \multicolumn{2}{|c|}{ Fonctions des répondants } \\
\hline \multirow{3}{*}{$\begin{array}{l}\text { Syndicats et } \\
\text { fédérations }\end{array}$} & Fédération Française des Syndicats du Liège & Chargé de mission technique et de promotion \\
\hline & $\begin{array}{l}\text { Syndicat des forestiers privés des } \\
\text { Pyrénées-Orientales }\end{array}$ & Ingénieur forestier \\
\hline & Union de la Coopération Forestière Française & Directeur technique \\
\hline \multirow{13}{*}{$\begin{array}{l}\text { Structures } \\
\text { collectives } \\
\text { régionales }\end{array}$} & \multirow{2}{*}{ Institut Méditerranéen du Liège } & Responsable de I'ASL Catalane \\
\hline & & Président \\
\hline & Association Le Liège Gascon & Chargée de mission \\
\hline & ASL Suberaie Varoise & Ingénieur forestier \\
\hline & ASL Catalane & Ingénieur forestier \\
\hline & \multirow{3}{*}{ Centre Régional de la Propriété Forestière } & Ingénieur forestier \\
\hline & & Technicien \\
\hline & & Ingénieur forestier \\
\hline & \multirow[t]{2}{*}{ Coopérative Corsica Furesta } & $\begin{array}{l}\text { Ancien président de la coopérative } \\
\text { et propriétaire forestier }\end{array}$ \\
\hline & & Ancien ingénieur forestier de la coopérative \\
\hline & \multirow{2}{*}{$\begin{array}{l}\text { Ecopole SIVOM Association Maures Bois } \\
\text { Énergie }\end{array}$} & Chargé de mission environnement \\
\hline & & Responsable SIVOM \\
\hline & Apcor & Président de l'association et industriel WSF Cork \\
\hline \multirow{12}{*}{ Industriels } & Agglolux-CBL & Dirigeant \\
\hline & Junque & Dirigeant \\
\hline & Melior & Dirigeant \\
\hline & Amorim France & Directeur général filiale France \\
\hline & Amorim & Responsable de la communication \\
\hline & Amorim & Responsable travail forestier \\
\hline & Bourrasse & Directeur commercial \\
\hline & Abel Bouchons & Dirigeant \\
\hline & Au liégeur & Dirigeant \\
\hline & Cork-Design & Dirigeant \\
\hline & Casabio & Dirigeant \\
\hline & Les Bouchons Corses & Dirigeant \\
\hline \multirow{5}{*}{ Propriétaires } & \multicolumn{2}{|l|}{ Propriétaire et industriel retraité } \\
\hline & \multicolumn{2}{|l|}{ Groupement Forestier de la Fouquette } \\
\hline & \multicolumn{2}{|l|}{ Groupement Forestier de l'Estérel } \\
\hline & \multicolumn{2}{|l|}{ Domaine des Campaux } \\
\hline & \multicolumn{2}{|l|}{ Porto-Vecchio } \\
\hline \multirow{3}{*}{ Experts } & \multicolumn{2}{|l|}{ Professeur d'Université en géographie } \\
\hline & \multicolumn{2}{|l|}{ Doctorant en histoire de la filière liège } \\
\hline & \multicolumn{2}{|c|}{ Architecte, ingénieur des Travaux Publics, propriétaire forestier } \\
\hline
\end{tabular}

Source : les auteurs. 
Ces stratégies collectives s'appuient le plus souvent sur des acteurs tiers, comme l'indiquent les cas de l'industrie cinématographique (Roy, 2004 ; Yami, Le Roy, 2006), de l'industrie de la conserve de poissons (Le Roy, 2003), des métiers d'art (Loup, 2003), ou encore du liège (Dari, 2013, 2014). Leur présence accroît la durée de vie des entreprises qui en sont partie prenante (Baum, Oliver, 1991), entreprises qui doivent, en contrepartie, adhérer explicitement à ses règles (Meyer, Rowan, 1977 ; Scott, Meyer, 1983). La stratégie collective induit cependant une sorte de zone de protection qui limite la compétition, et parfois la volonté d'innovation d'entreprises se sentant à l'abri au sein de l'écosystème (Le Roy, 2003). Il est donc nécessaire que l'acteur tiers stimule les échanges entre partenaires afin de créer des synergies au sein de la filière.

\section{Des stratégies collectives gagnantes}

Identifier les bonnes pratiques managériales mises en place dans les régions Aquitaine, Pyrénées-Orientales et Var permet d'avoir une vision globale des projets conduits de manière efficace et ayant obtenu des résultats probants pour relancer la filière liège. Les bonnes pratiques renvoient à la manière dont s'organise l'acteur tiers et à son influence réelle dans ladite relance de la filière, en référence à ses objectifs, rôles et missions. S'il n'est pas possible d'évaluer les bonnes pratiques d'un point de vue quantitatif, certains éléments permettent toutefois de valider positivement leurs actions, à travers les retours des différents acteurs interrogés, l'aboutissement de projets, la «longévité » des structures ou encore la reconnaissance par les acteurs de la filière. La recherche de terrain conduit à observer cinq types de structures collectives ayant pour principal objectif le maintien d'une activité liège. Deux structures sont nationales et trois structures sont régionales (voir le tableau 2) :
- Deux structures nationales : la Fédération Française des Syndicats du Liège, qui se focalise essentiellement sur les entreprises de bouchons ; l'Institut Méditerranéen du Liège, qui a pour vocation d'être un lieu de recherche et de développement dédié à toutes les activités de la filière.

- Trois structures régionales : 1'Association Le Liège Gascon, qui réunit principalement des industriels, regroupement unique aujourd'hui en France dans la mesure où les structures représentent traditionnellement des propriétaires forestiers ; les ASL (Associations Syndicales Libres) du Var et des Pyrénées-Orientales, qui sont des associations représentant les propriétaires forestiers.

La synthèse des approches de l'acteur tiers en sciences de gestion a permis d'identifier quatre dimensions clés du succès de ces acteurs. Il est tout d'abord un acteur neutre garantissant un niveau de confidentialité élevé au profit des acteurs dès l'instant où ils s'associent à la structure porteuse d'une stratégie collective pour la filière. Il s'agit ensuite d'une structure fédératrice et représentative de la filière dans son ensemble, construisant un réseau relationnel et de puissantes synergies entre acteurs. L'acteur tiers a également pour vocation d'être un support de compétences et de connaissances, tout en assurant un rôle de contrôle et de sanction lorsque les règles du jeu ne sont pas respectées par l'un des acteurs. Enfin, il est le représentant du réseau en créant du lien et en développant des projets au sein de la filière (voir le tableau 3). En ce sens, l'acteur tiers se présente comme un substitut possible à la coordination verticale par le contrat, qui se présente habituellement comme une voie d'amélioration de la compétitivité d'une filière, notamment par une redistribution équitable de la valeur aux différents acteurs (Bouamra-Mechemache et al., 2015). 


\begin{tabular}{|c|c|c|c|}
\hline 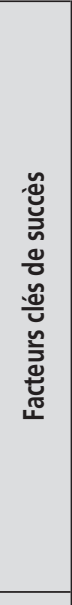 & 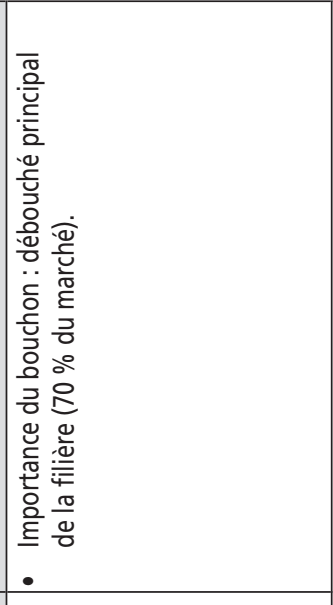 & 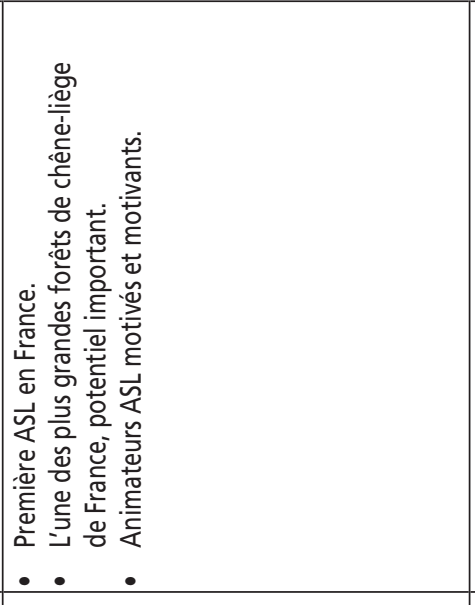 & 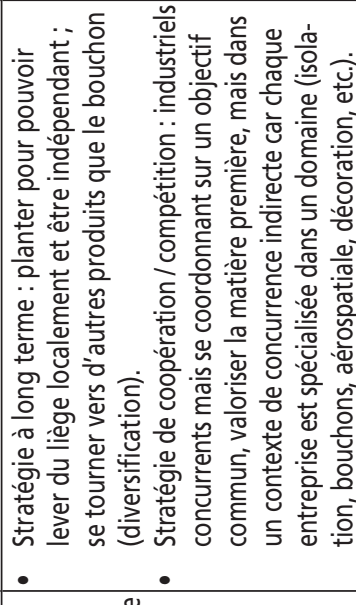 \\
\hline 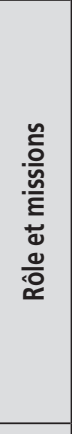 & 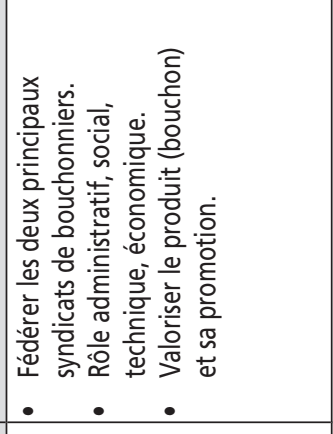 & 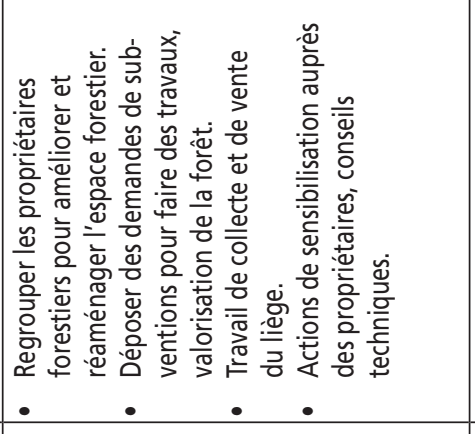 & 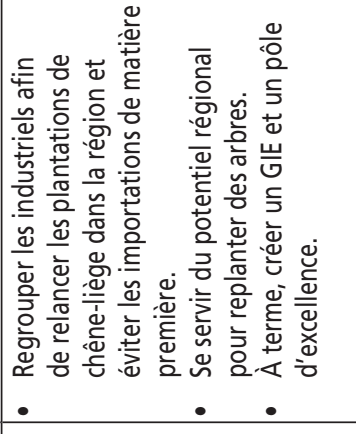 \\
\hline 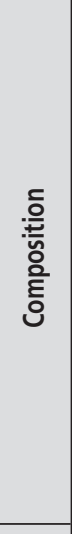 & 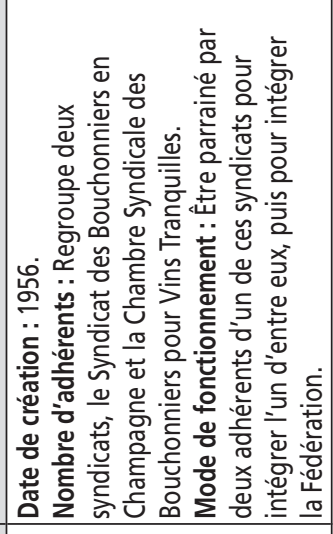 & 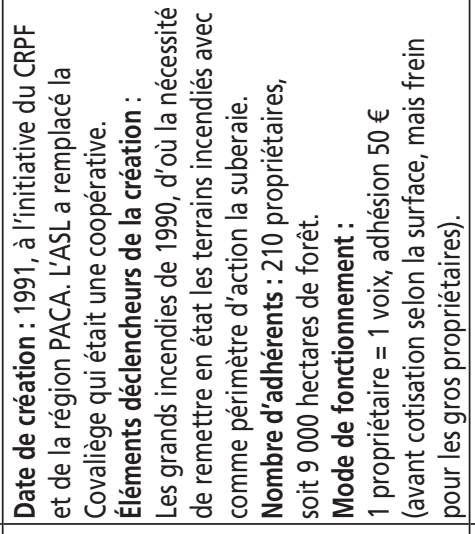 & 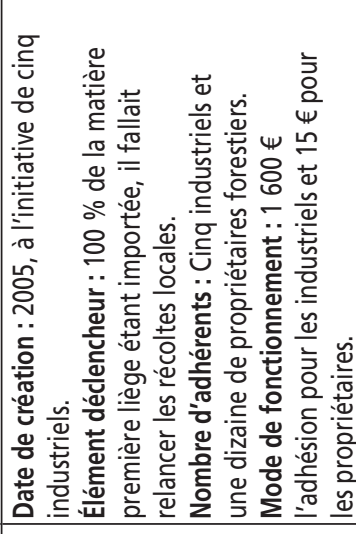 \\
\hline 은 & $\begin{array}{l}\overline{\widetilde{0}} \\
\stackrel{0}{0} \\
\text { त0 }\end{array}$ & $\stackrel{\frac{1}{>}}{>}$ & 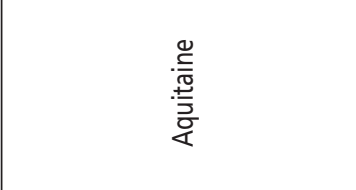 \\
\hline 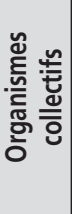 & 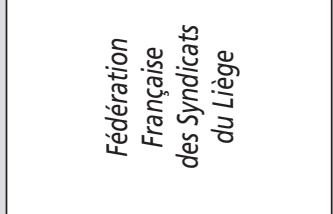 & 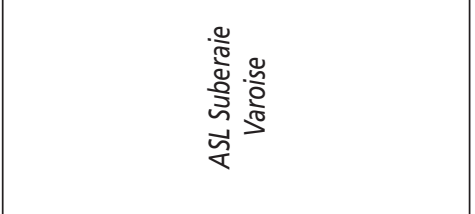 & 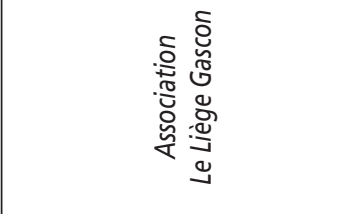 \\
\hline
\end{tabular}




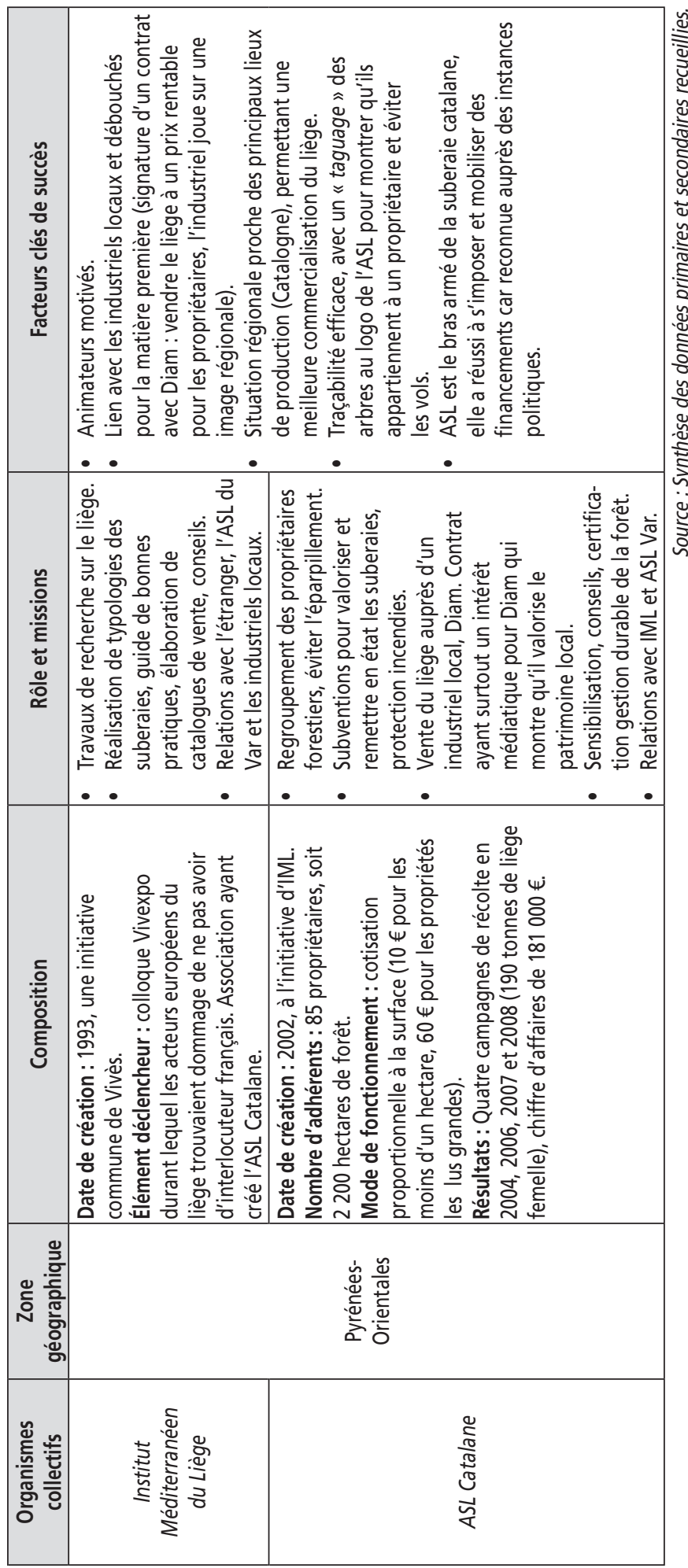


Ses deux principales missions, pour reprendre Xhauflair et Pichault (2012), sont ainsi de penser un cadre renouvelé pour la coordination et d'initier un processus continu d'essais / erreurs :

- Penser un cadre renouvelé pour la coordination. L'acteur tiers conduit un travail cognitif de description de chaînes de causalité en contribuant à créer un cadre ad hoc au sein duquel les acteurs du collectif formalisent de nouveaux schémas d'interaction.

- Initier un processus continu d'essais / erreurs. L'acteur tiers teste différents registres d'action et suggère des ajustements pour intégrer les attentes des uns et des autres, une démarche interactive qui confronte progressivement les acteurs à la réalité de terrain.

Malgré les apports à mettre à l'actif des acteurs tiers en matière de coordination et de processus essais / erreurs, des difficultés ont pu être identifiées par les répondants lors des entretiens menés dans les régions Aquitaine, Pyrénées-Orientales et Var, parmi lesquelles : réussir à financer des levées de liège mâle et négocier des prix avantageux pour les propriétaires ; sensibiliser et communiquer sur les atouts du produit ; assurer un contrôle des activités en forêt afin notamment d'éviter les vols. Il n'en demeure pas moins que la présence de structures collectives dans ces trois régions françaises possédant du liège est porteuse d'une forte dynamique, ayant favorisé le maintien à la fois des suberaies et des savoir-faire ancestraux leur étant attachés. Bouisset et Puyo (2011) relatent ainsi comment un processus de « moralisation », pour empêcher abus et dérives, au premier rang desquelles des levées de liège trop précoces, y a conduit à des ventes groupées de liège aux enchères, mais aussi la création de l'association internationale Retecork (réseaux des territoires du liège) fédérant acteurs publics et privés partageant un intérêt pour le liège.

\section{Tableau 3. Les quatre rôles de l'acteur tiers}

\begin{tabular}{|c|c|}
\hline RÔLE DE NEUTRALITÉ & RÔLE DE FÉDÉRATEUR ET DE REPRÉSENTATIVITÉ \\
\hline $\begin{array}{l}\text { - Regrouper les acteurs importants et arriver } \\
\text { à intéresser les plus petits. } \\
\text { - Parler d'une seule voix. }\end{array}$ & $\begin{array}{l}\text { - Fédérer autour d'un objectif commun : garantir } \\
\text { les aspects traditionnels et patrimoniaux du liège, } \\
\text { et trouver des débouchés. } \\
\text { - Négocier, être un acteur qui pèse. } \\
\text { - Développer une synergie de moyens, de } \\
\text { connaissances et de compétences, mutualiser } \\
\text { des moyens. } \\
\text { - Établir un tissu relationnel. } \\
\text { - Sensibiliser et communiquer auprès du grand } \\
\text { public et des prescripteurs (État, BTP, architectes, } \\
\text { etc.). }\end{array}$ \\
\hline RÔLE DE PILOTAGE ET D'ANIMATION & RÔLE DE CONTRÔLE ET DE SANCTION \\
\hline $\begin{array}{l}\text { - Créer des liens et un espace d'échange entre } \\
\text { les acteurs. } \\
\text { - Anticiper l'avenir, réflexion à l'échelle du } \\
\text { territoire, stratégie de développement } \\
\text { économique. } \\
\text { - Questionner, débattre, échanger, force de } \\
\text { propositions. } \\
\text { - Se regrouper pour résoudre collectivement } \\
\text { les problèmes. }\end{array}$ & $\begin{array}{l}\text { - Conseiller, diagnostiquer, valoriser la forêt, faire } \\
\text { - un état des lieux des peuplements. } \\
\text { le travail et faire gagner du temps, délégation, } \\
\text { apporter un service et une prise en charge. } \\
\text { - Améliorer la connaissance de la législation, } \\
\text { des compétences techniques et juridiques. } \\
\text { - Développer des réunions d'information et } \\
\text { de sensibilisation. }\end{array}$ \\
\hline
\end{tabular}

Source : Adapté de Greenwood et al. (2002). 
Dans ces trois régions, les structures collectives se sont avérées efficaces, même si la production de liège reste dépendante de fluctuations de cours non négligeables.

Les enquêtes avec les acteurs au sujet des facteurs contribuant à ce succès des acteurs tiers corroborent ceux identifiés dans la revue de la littérature (notamment à partir des travaux de Greenwood et al., 2002) : neutralité de la structure assurant une représentation équitable de tous les membres ; fédération de l'ensemble des acteurs du secteur ; pilotage des actions de relance et communication pour le compte du collectif ; contrôle et définition de règles du jeu afin de stabiliser les actions individuelles et de contenir les comportements opportunistes. La présence d'acteurs tiers et leurs actions continues de soutien ont permis de garantir la survie de la filière. Si des difficultés sont rencontrées, et si certaines missions doivent être menées de manière plus efficace, l'acteur tiers reste malgré tout un véritable pivot générateur de registres d'action nouveaux, et reconnu comme tel. Le cas du liège en Corse semble, de ce point de vue, profondément atypique.

\section{Le cas singulier du liège en Corse}

En première approximation, la filière liège en Corse semble avoir pour particularités une qualité supérieure, des débouchés abondants et une ressource sous-utilisée, voire laissée à l'abandon. Un examen plus attentif indique cependant que la ressource essentielle, à savoir le liège femelle, est rare car les suberaies ne sont plus exploitées, comme elles le sont par exemple au Portugal. Actuellement à l'abandon, le liège doit donc être retravaillé pour être de bonne qualité, et s'assurer des débouchés suffisants. Ces derniers pourraient d'ailleurs s'avérer abondants, mais à la condition qu'une activité industrielle et commerciale soit mise en place pour conquérir des marchés, ce qui n'est pas le cas. En d'autres termes, nous sommes face à des potentialités du territoire et leur réelle expression passe sans doute par une démarche coordonnée et volontariste de la part du collectif d'acteurs. Or notre investigation conduit à constater l'absence de structure fédérative en Corse fondée sur l'action d'un acteur tiers. Il n'existe ainsi aucun ASL, institut, syndicat ou fédération œuvrant spécifiquement au sein de la filière du liège pour accroître sa valorisation.

Il a toutefois été possible d'identifier la présence d'une coopérative, Corsica Furesta, dissoute en mars 2013. La coopérative avait été mise en place à l'initiative de propriétaires forestiers en décembre 2009. Elle regroupait 28 propriétaires disposant de surfaces entre 3 et 1700 hectares. Une cotisation à l'entrée dans la coopérative de $15 € /$ hectare était prévue. Outre le classique soutien des propriétaires en termes de compétences opérationnelles et de conseils pour gérer au mieux les forêts et les remettre en état, la création de la coopérative avait trois objectifs :

- Relancer les activités autour de la suberaie corse. Il existe $75 \%$ de milieu dit boisé, avec un potentiel productif proche de celui des années 1920, soit 10000 tonnes de liège (après remise en état des forêts et remise en culture). Aucun acteur économique privé n'étant suffisamment solide financièrement pour lancer des opérations d'envergure, il était pertinent de mettre collectivement à profit le potentiel existant.

- Bénéficier des potentialités du marché. D'une part, le liège fait partie du patrimoine culturel de l'île et constitue une matière première de qualité (absence du ver du liège, climat favorable pour son développement). D'autre part, la proximité géographique avec la Sardaigne permet de disposer de la présence d'acteurs compétents pour la transformation de la matière première et d'importants débouchés commerciaux. Enfin, il s'agit d'une 
matière naturelle et pour laquelle il existe une demande et de nouveaux débouchés ; ce qui rendait sa relance cohérente.

- Définir un cadre et des règles. La levée du liège fait partie d'un système opaque où la négociation des prix varie sensiblement d'un acteur à l'autre (Dari, 2014). Par le passé, le manque de transparence a souvent entraîné des abus. Des leveurs de liège peu scrupuleux n'hésitaient pas à négocier le prix de la matière première à la baisse, notamment auprès des propriétaires, qui n'avaient aucune connaissance et compétence dans le domaine. L'absence de structure jouant le rôle de référent ne permettait donc pas de garantir un rapport équilibré entre acteurs, d'où l'alternative d'un regroupement de propriétaires.

Néanmoins, la coopérative Corsica Furesta a dû faire face à différents types de contraintes l'empêchant de s'établir durablement. Des problèmes internes récurrents ne lui ont pas permis de se structurer et de définir correctement ses missions ; le résultat en fut des difficultés récurrentes de gestion, des conflits de personnes, ou encore un ingénieur forestier embauché bien trop tardivement (voir le tableau 4). Dans ces conditions, les rôles de fédérateur, d'animateur et de contrôleur devant être tenus par l'acteur tiers n'ont pas pu être instaurés. À quoi s'ajoute une incapacité manifeste à exploiter les potentialités du marché faute d'une véritable démarche commerciale. Les acteurs n'ayant pas souhaité intégrer la coopérative ont poursuivi leurs actions individuelles tout en œuvrant pour empêcher la signature de contrats d'approvisionnement entre la coopérative corse et les industriels sardes. Seule une levée de liège a pu être effectuée sur quatre propriétés, dans le cadre d'un contrat d'approvisionnement avec le leader portugais Amorim. Circonstance aggravante, l'absence de soutien financier de la part de la classe politique, soutien que l'on peut observer à l'inverse dans les autres régions françaises, n'a pas permis d'assurer la pérennité de la coopérative.

La mise en place d'une convention coopérative s'appuie en outre sur un certain nombre de contraintes. Ces contraintes de production doivent être respectées par la coopérative durant une période donnée. Dans le cas de Corsica Furesta, il avait été prévu de lever 1000 tonnes de liège, 8 tonnes de plaquettes forestières, $500 \mathrm{~m}^{3}$ de châtaignier bois d'œuvre, d'effectuer 5 plans simples de gestion (PSG) et 3 règlements types de gestion (RTG). Or seulement $48 \%$ de l'objectif a été rempli par Corsica Furesta. La convention coopérative était en effet démesurée par rapport au temps imparti et ne pouvait pas être correctement effectuée sans la présence d'un ingénieur forestier. Fondée en 2009, la coopérative n'a pu seulement débloquer des fonds qu'en 2011 pour recruter cet ingénieur. L'absence d'aides financières, pourtant prévues au départ du projet, et d'un expert pour atteindre les objectifs a entraîné progressivement la dissolution de la coopérative au début de l'année 2013. Les contraintes économiques, liées au type de structure, ne lui ont pas permis d'être aussi souple qu'une structure de type associatif. Ceci est sans doute dû à la prééminence d'une logique asymétrique selon laquelle les plus importants opérateurs ont le sentiment de contribuer à la croissance des plus petits. Les entretiens font ressortir que des difficultés liées à l'environnement local dans lequel se trouvait la coopérative (spécificités culturelles et historiques du territoire insulaire) ont rendu la situation plus qu'incertaine :

- L'indivision en Corse ne permet pas toujours de connaître les propriétaires des terrains et entraîne souvent un morcellement des parcelles entre plusieurs héritiers. Étant donné que $77 \%$ des suberaies sont privées (Inventaire Forestier National, 2009), leur remise en état ne 


\begin{tabular}{|c|c|}
\hline RÔLE DE NEUTRALITÉ & RÔLE DE FÉDÉRATEUR ET DE REPRÉSENTATIVITÉ \\
\hline $\begin{array}{l}\text { - Problème du clientélisme, mauvais } \\
\text { positionnement politique des derniers membres } \\
\text { du bureau, ce qui ne leur a pas permis d'obtenir } \\
\text { une aide financière. } \\
\text { - Présence de deux acteurs économiques dans la } \\
\text { coopérative : I'ODARC et le CRPF. En dehors des } \\
\text { conflits de personnes existants entre ces deux } \\
\text { organismes, la coopérative n'avait pas de réelle } \\
\text { indépendance par rapport aux actions qu'elle } \\
\text { devait mener. }\end{array}$ & 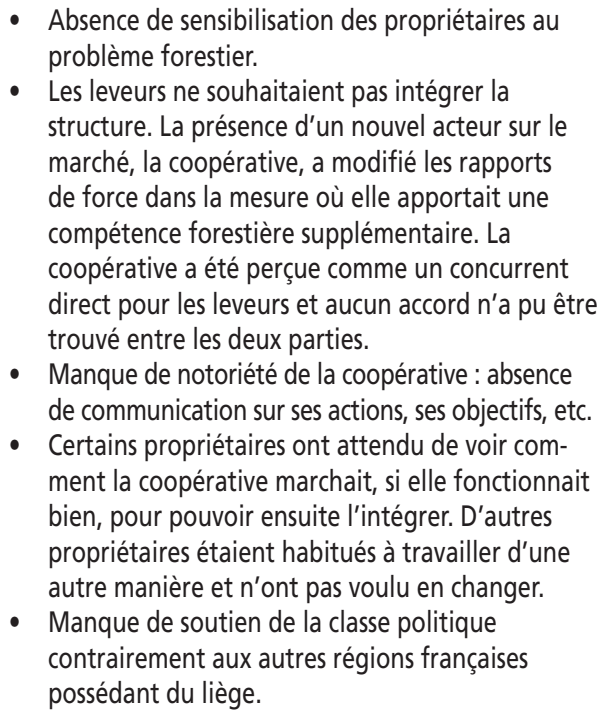 \\
\hline RÔLE DE PILOTAGE ET D'ANIMATION & RÔLE DE CONTRÔLE ET DE SANCTION \\
\hline $\begin{array}{l}\text { - Problème de conflits interpersonnels entre les } \\
\text { responsables: désaccord sur la manière dont } \\
\text { la coopérative devait être gérée, } 3 \text { présidences } \\
\text { en moins de } 4 \text { ans, pas de pilote représentatif. } \\
\text { Constitution de la coopérative autour de gens } \\
\text { ayant des difficultés à travailler ensemble. } \\
\text { - Division entre les propriétaires du Nord (bois de } \\
\text { chauffage) et du Sud (liège). } \\
\text { - Absence d'objectifs communs, partagés par tous, } \\
\text { pas de vision commune du projet. } \\
\text { Mauvaise gestion financière de la coopérative, } \\
\text { prise de décision individuelle. } \\
\text { La coopérative a eu des difficultés à obtenir un } \\
\text { contrat de négociation avec les industriels sardes, } \\
\text { déjà en contrat avec les leveurs existants. } \\
\text { Inertie dans la prise de décisions, manque de } \\
\text { motivation et de synergies, difficultés à mobiliser } \\
\text { les acteurs. }\end{array}$ & $\begin{array}{l}\text { - Problème du contrôle des parcelles : certains } \\
\text { leveurs (corses ou sardes) profitent de collecter } \\
\text { du liège sur les parcelles voisines sans autorisation } \\
\text { du propriétaire, d'où des vols importants de liège } \\
\text { et de bois. } \\
\text { - Un seul ingénieur pour des surfaces importantes. } \\
\text { De plus, celui-ci n'a été embauché qu'en } \\
\text { janvier } 2011 \text { et n'est resté que } 16 \text { mois. Son } \\
\text { arrivée tardive et temporaire ne permet pas de } \\
\text { développer des projets à long terme. }\end{array}$ \\
\hline
\end{tabular}

Source : Synthèse des données primaires et secondaires recueillies.

peut donc pas se faire sans l'accord du (ou des) propriétaire(s).

- L'abandon des suberaies a entraîné une diminution de la qualité du liège. La matière première n'est pas tout de suite rentable et ne le sera que d'ici 20 à 30 ans. Les propriétaires n'ayant pas une vision à long terme, ils n'ont pas toujours, voire rarement, la motivation pour investir dans leur suberaie.

- Le problème récurrent de partage de l'espace forestier avec les agriculteurs et les éleveurs conduit au pâturage des animaux en divagation, empêchant ainsi la régénération des suberaies dans des conditions idéales. 
- Enfin, d'une manière plus générale, le manque de confiance des investisseurs étrangers dans le devenir de l'économie corse, compte tenu de l'instabilité de l'environnement institutionnel, ne permet pas toujours de convaincre des industriels continentaux de s'y installer.

De par sa mauvaise organisation interne, Corsica Furesta a ainsi progressivement perdu sa légitimité, en n'adoptant pas le comportement souhaitable compte tenu des normes sociales en présence, mais en faisant aussi face à un environnement externe particulièrement instable. L'expérience conduite ne remet cependant pas en question l'intérêt d'un organisme collectif de type tiers acteur pour coordonner un secteur. Des enseignements doivent au contraire en être tirés afin de parvenir à une situation plus satisfaisante dans la mesure où l'histoire semble bégayer. En effet, une nouvelle coopérative, dénommée A Silva, a été créée fin 2013, et rencontre des difficultés similaires à celles de Corsica Furesta. Sans en tirer de conclusion hâtive, il semblerait ainsi que le format de la coopérative ne soit pas adapté à la relance d'une filière où les positions concurrentielles sont très différentes entre acteurs. Le cas de la race porcine corse dans un projet AOP, étudié par LambertDerkimba et al. (2011), conforte l'idée que de nombreuses tensions existent entre les gestionnaires d'une ressource et les acteurs économiques qui cherchent à la mobiliser dans leur projet.

Le cas corse témoigne de la persistance de stratégies purement individuelles qui ne permettent pas une véritable relance de l'activité. Le système demeure opaque et les acteurs ne se projettent pas à long terme. Il se démarque de façon nette des autres régions où la volonté de travailler collectivement, avec ou sans acteur tiers, permet de développer des projets communs allant dans le sens d'une reconstruction de la filière liège. Même si l'acteur tiers y fait parfois l'objet d'une réelle défiance (comme dans le Var, où l'industriel travaille peu avec l'ASL, représentant des propriétaires), le territoire continue pourtant à avancer. Les syndicats et fédérations exercent une action significative au niveau national et se focalisent principalement sur un seul produit issu du liège : le bouchon. L'absence ou la présence d'un acteur tiers efficace n'est donc pas nécessairement une condition de succès des actions collectives, mais il constitue l'un des éléments d'analyse permettant de mieux comprendre l'hétérogénéité des formes inter-organisationnelles et les logiques territoriales en œuvre dans les filières.

\section{Discussion}

Le recours à une étude de cas permet de tester certains éléments identifiés dans la théorie managériale comme les garants du succès d'un acteur tiers : neutralité, représentativité, animation et contrôle, et de les confronter à d'autres éléments contextuels. Les aspects culturels liés aux acteurs composant l'écosystème (filière) sont essentiels pour comprendre la possible réussite d'une stratégie collective. Si l'acteur tiers se présente comme une composante importante de la relance d'une filière, le cas du liège en Corse illustre le fait qu'il n'est pas toujours perçu comme nécessaire par les acteurs. Ces derniers ne voient pas l'intérêt de la mise en œuvre d'une logique d'action collective pouvant, le cas échéant, contrarier leurs projets individuels. Nous retrouvons ici plusieurs des éléments identifiés par le passé pour expliquer l'échec de la dynamique collective (Bresser, Harl, 1986 ; Le Roy, 2003 ; Yami, 2003 ; Granata, 2010 ; Loubaresse, Pestre, 2014) : des intérêts individuels trop prégnants ne permettant pas de faire émerger une dynamique commune; l'échec des missions assumées par l'acteur tiers, conduisant à la remise en cause de son rôle de coordonnateur ; des conflits d'intérêt 
entre les acteurs, notamment au niveau de la répartition de la rente ; une absence d'identité collective et d'objectifs (voire de valeurs) partagés par tous.

Il est en effet important que l'ensemble des acteurs adoptent un état d'esprit commun, partageant la même vision du projet, avec une réelle motivation pour la relance de l'activité, ici du liège. Autrement dit, les aspects économiques ne doivent pas tout de suite régir les fondements de la structure collective, d'autant plus que le liège n'est pas un produit immédiatement rentable. La lisibilité du projet doit être autant économique que sociale (Chomel et al., 2014). À ce titre, le choix du type d'organisation incarnant l'acteur tiers (coopérative ou association, par exemple) selon le contexte de la filière semble être crucial. Deux autres aspects nous semblent également importants à souligner :

- Il existe un effet de dépendance perçue des acteurs à la structure collective en termes de mise en contact avec les marchés et de connaissance des attentes de la clientèle. Souvent évoquée lors des entretiens, la dépendance apparaît comme un élément pouvant freiner la volonté collaborative les acteurs. Dans ces conditions, il semble essentiel que la structure collective dispose de compétences réelles et propose une vraie valeur ajoutée pour ses adhérents.

- Les acteurs de la filière peuvent considérer la structure collective comme un moyen astucieux de profiter de financements pour réhabiliter leur forêt et $\mathrm{y}$ faire des travaux, bénéficiant aussi des compétences techniques de l'acteur tiers. Une démarche visant à intégrer durablement ces acteurs, afin d'éviter toute forme d'opportunisme de leur part, doit tenir compte du problème de dépendance précédemment évoqué.

Il est ici possible de faire le lien entre la notion d'acteur tiers et le concept de bro$k e r$, et d'en suggérer une application à la filière du liège (voir le tableau 5). Un broker a pour objectif de coordonner les entreprises qui font partie d'un réseau d'affaires (Miles, Snow, 1986 ; Snow et al., 1992 ; Loubaresse, 2008). Le broker peut être considéré comme un pivot, c'est-à-dire une structure qui domine le réseau (Jarillo, 1988), ou comme un courtier jouant le rôle d'intermédiaire entre les entreprises (Boyle, 1994 ; Lecocq, 2003 ; Cappetta, Cillo, 2008). Snow et al. (1992) identifient trois types de brokers, à savoir l'architecte, le coordinateur et le facilitateur, qui trouvent un écho direct dans la filière du liège :

- L'architecte favorise les relations entre entreprises et facilite la constitution du réseau dans la mesure où il dispose d'une vision globale de celui-ci. Dans le cas de la filière du liège, l'acteur tiers, compte tenu de sa position centrale, va fédérer un certain nombre d'adhérents autour d'un projet commun; il s'agit là de sa mission principale. Les ASL varoise et catalane fédèrent ainsi les propriétaires forestiers afin de remettre notamment en état les suberaies et trouver des débouchés pour le liège levé. L'Association Le Liège Gascon est, quant à elle, un bel exemple d'acteur tiers architecte dans la mesure où elle regroupe à la fois des propriétaires et des industriels. La structure parle ainsi d'une seule voix et représente l'ensemble des acteurs de la filière. Sa légitimité découle de sa capacité à représenter de manière unanime et neutre les adhérents.

- Le coordinateur est garant de l'efficience du réseau en coordonnant les compétences et les objectifs des entreprises pour faciliter la conquête des marchés. Dans le cas des structures collectives étudiées au sein de la filière du liège, ces dernières assurent le pilotage des acteurs de leur réseau. Elles sont en quelque sorte les chefs d'orchestre du collectif et se coordonnent entre elles afin 
d'échanger sur leurs expériences respectives : en animant les projets (création d'un pôle de broyage bois / liège afin de créer de nouveaux débouchés), en créant des synergies entre acteurs (contrat d'approvisionnement entre les ASL varoise et catalane et un industriel, conférences et ateliers de travail), et en faisant bénéficier tous les membres de leurs compétences clés (techniques, conseils, etc.).

- Le facilitateur, enfin, s'assure de la durabilité du réseau en diffusant l'information entre les entreprises. La position occupée permet de contrôler d'éventuelles déviances et d'apporter des corrections pour que la valeur que chaque acteur apporte ne diminue pas par rapport aux autres, voire que certains acteurs n'exploitent pas le réseau pour obtenir des gains individuels à court terme. Force est d'admettre qu'il est pour le moment prématuré d'identifier une structure collective ayant une fonction de facilitateur dans la filière du liège. La plupart des structures existantes sont récentes et commencent seulement à regrouper, fédérer et concevoir des projets collectifs viables. De plus, si elles parviennent à réunir des acteurs, elles ne sont pas encore en capacité de trouver des débouchés rentables et de les faire travailler ensemble.

Il ressort des entretiens conduits que chaque structure est pour le moment amenée à représenter une seule catégorie d'adhérents faisant partie de la filière (les propriétaires forestiers, les bouchonniers / les industriels, les fabricants d'isolants, etc.), mais sans qu'aucun lien ne vienne se créer entre eux. La vision est cloisonnée ; elle repose sur des optimisations locales, indépendamment d'un hypothétique optimum global dont les gains pourraient être collectivement partagés. Ceci n'est pas réellement surprenant, notamment si l'on examine dans une perspective historique le fonctionnement des chaînes logistiques multi-acteurs, qui peuvent également s'apparenter à des écosystèmes. Tixier et al. (1996) ont ainsi montré que les méthodes logistiques les plus modernistes se sont d'abord développées pour accroître la performance de chaque maillon indépendamment les uns des autres (amélioration du transport, rationalisation des opérations de manutention, etc.). Il faudra de nombreuses années pour qu'une vision globale et systémique l'emporte, en mettant en lumière l'importance d'une vision collective pour améliorer la performance coût-service, ce qui n'est pas le cas pour la filière du liège en Corse.

$$
\begin{gathered}
* \\
* \quad *
\end{gathered}
$$

Acteur de la gouvernance des relations inter-organisationnelles, l'acteur tiers se positionne comme médiateur, capable d'établir un lien entre les entreprises d'une filière pour améliorer leur performance. Acteur également neutre, garant de la représentativité de tous les acteurs, il assure un pilotage des actions menées en canalisant la logique d'action collective. Ce rôle d'animateur permet de faire bénéficier les adhérents de compétences et de connaissances spécifiques, tout en créant un espace relationnel et des effets de synergie entre eux. Une telle dynamique collective est ainsi un élément essentiel pour améliorer la compétitivité de la filière. Progressivement, la légitimité d'expertise acquise par l'acteur tiers devient un moyen d'assumer une mission de dynamisation et de contrôle dans la filière, mais aussi de sanction si les règles du jeu ne sont pas respectées par tous. Une légitimité pragmatique, au sens de Suchman (1995), découlant de la capacité de l'acteur tiers à répondre efficacement à des intérêts particuliers dans le cadre de registres communs d'action, s'affirme progressivement. L'organisme collectif devient le garant de la bonne marche de la filière, et sa neutralité permet de contourner le problème récurrent de la clarification des droits de propriété 


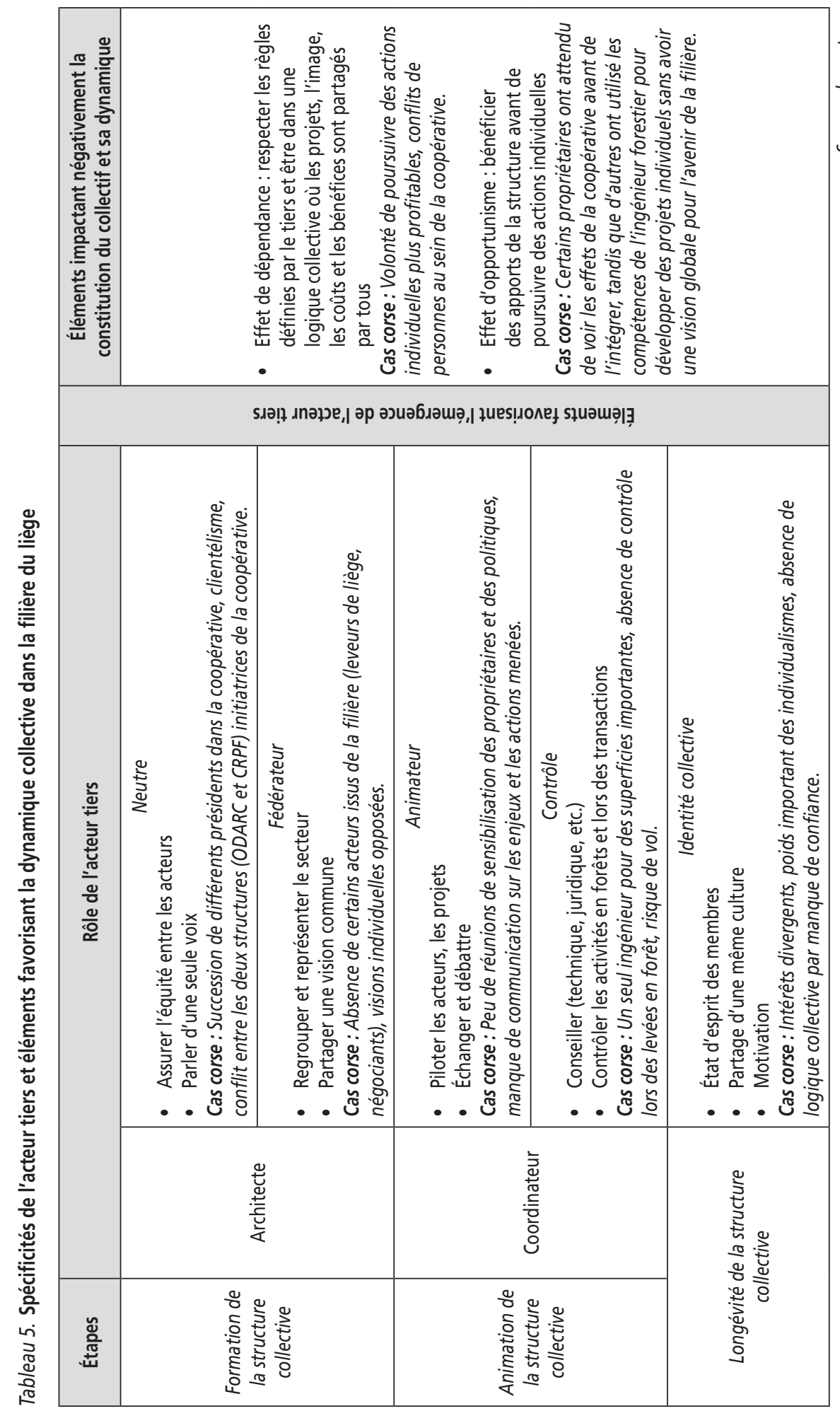


sur la rémunération de chaque partenaire, problème particulièrement sensible dans les coopératives (Chomel et al., 2014).

Les réalités contrastées qui émergent du cas du liège en France soulignent finalement l'actualité des approches en termes de filière. Dans de nombreux travaux, la filière doit d'abord être vue comme la succession des étapes technologiques de production distinctes et séparables, associées à l'utilisation d'une ressource donnée ou à l'utilisation d'un produit déterminé (Soulié, 1980). Une telle utilisation a pu limiter la portée analytique du concept de filière. Même si Pérez (1983) a souligné très tôt l'importance des stratégies d'entreprises conduites au sein de l'écosystème « filière », la filière a été trop souvent réduite à un artefact conceptuel qui « reconstruit » la réalité d'un agencement de séquences au sein de systèmes industriels. Pourtant, dans sa capacité à représenter, selon une logique méso-économique, la manière dont des acteurs complémentaires interagissent (ou non) entre eux, elle est d'une pertinence incontestable. En plaçant au cœur de l'analyse les notions de domination et de captation de la valeur (Sekkat, 1996), les approches en termes de filière soulignent les effets des tensions qui se manifestent, de manière récurrente, dans le management des relations interorganisationnelles.
L'étude de la filière du liège en France enrichit ainsi les connaissances au sujet des dynamiques alternant coopération et confrontation. Elle indique que l'organisme collectif chargé d'établir un dialogue constructif entre acteurs ne remplit pas toujours le rôle qui lui était dévolu à l'origine. C'est tout particulièrement le cas de la Corse. Cette situation singulière s'explique tout d'abord par le choix de la structure, une coopérative, dont certaines caractéristiques (rigidité des statuts, obligations de production, etc.) semblent peu compatibles avec les conditions requises pour assurer une intermédiation efficace, particulièrement en situation d'asymétrie concurrentielle. L'étude de cas souligne également l'importance des facteurs environnementaux externes, liés à des facteurs culturels, historiques ou politiques propres à la région dans laquelle la coopérative s'est insérée. Enfin, parmi les éléments clés évoqués précédemment pour garantir le succès de l'intermédiation, aucun n'a été correctement mis en place, notamment en termes de neutralité et de représentativité des acteurs. Une telle situation d'échec n'est pas rédhibitoire. Le retour d'expérience qu'elle autorise peut être le point de départ de l'établissement d'une nouvelle logique collective, portée par un ordre institutionnel différent, permettant d'envisager à terme une relance durable de la filière du liège.

\section{RÉFÉRENCES BIBLIOGRAPHIQUES}

Aoki M. (2001). Toward a comparative institutional analysis. Cambridge (MA), MIT Press.

Apcor (2006). Liège. Santa Maria de Lamas, Association portugaise du Liège.

Astley W., Fombrun C. (1983). Collective strategy: social ecology of organizational environments. Academy of Management Review, vol. 8, n 4, pp. 576-587.

Bardin L. (2013). L'analyse de contenu. Paris, Presses universitaires de France, $2^{\mathrm{e}}$ éd.

Baum J., Oliver C. (1991). Institutional linkages and organizational mortality. Administrative Science Quarterly, vol. 36, n 3, pp. 187-218. 
Bessy C., Favereau O. (2003). Institutions et économie des conventions. Cahiers d'Économie Politique, $\mathrm{n}^{\circ}$ 44, pp. 119-164.

Botey J. (2012). La suberaie européenne : histoire, situation, marché et nécessité de la défendre auprès de l'Union européenne. Forêt méditerranéenne, vol. 33, $\mathrm{n}^{\circ}$ 1, pp. 31-38.

Bouamra-Mechemache Z., Duvaleix-Tréguer S., Ridier A. (2015). Contrats et modes de coordination en agriculture. Économie rurale, $\mathrm{n}^{\circ} 345$, pp. 7-28.

Bouisset C., Puyo J.-Y. (2011). Déprise rurale, incendies et patrimonialisation : comment sauver les suberaies roussillonnaises ? Annales de Géographie, n 677, pp. 88-99.

Boyer R. (2003). Les institutions dans la théorie de la régulation. Cahiers d'Économie Politique, $\mathrm{n}^{\circ}$ 44, pp. 79-101.

Boyle E. (1994). Managing organizational networks in Britain: the role of the caretaker. Journal of General Management, vol. 19, $\mathrm{n}^{\circ}$ 4, pp. 13-23.

Bresser R., Harl J. (1986). Collective strategy: vice or virtue? Academy of Management Review, vol. 11, n² 2, pp. 408-427.

Cappetta R., Cillo P. (2008). Managing integrators where integration matters: insights from symbolic industries. International Journal of Human Resource Management, vol. 19, $\mathrm{n}^{\circ} 12$, pp. 2235-2251.

Chomel C., Declerck F., Filippi M., Frey O., Mauget R. (2014). Coopératives agricoles : identité, enjeux et défis. Économie rurale, $n^{\circ} 344$, pp. 75-84.

Dari L. (2013). Relancer une activité locale par les stratégies collectives : le cas de la filière liège en Corse. Revue Forestière Française, vol. $65, \mathrm{n}^{\circ} 2$, pp. 183-200.

Dari L. (2014). Rapport d'étude sur la filière liège en Corse. État des lieux et perspectives d'évolution. Étude réalisée pour le GIP Corse Compétences.

Davis L., North D. (1971). Institutional change and American economic growth. Cambridge, Cambridge University Press.

Dugelay A. (1952). La production et l'industrie du liège en France. Revue Forestière Française, $\mathrm{n}^{\circ}$ 11, pp. 728-751.

Fourcade C. (2006a). Les systèmes agroalimentaires localisés : des stratégies de développement local originales ? Actes du $23^{e}$ Colloque Annuel du Conseil Canadien des PME et de l'Entrepreneuriat. TroisRivières (Québec), pp. 1-16 (CD-rom).

Fourcade C. (2006b). Entrepreneuriat collectif et petite entreprise : tous pour un ou un pour tous ? In Fourcade C., Paché G., Pérez R. (dir.), La stratégie dans tous ses états. Caen, Éditions Management \& Société, pp. 339-352.

Fourcade C., Muchnik J., Treillon R. (2010). Coopérations, territoires et entreprises agroalimentaires. Versailles, Quae.

Gadille M. (2008). Le tiers comme agent de réflexivité et accélérateur d'apprentissages collectifs : le cas du dispositif des pôles de compétitivité. Humanisme \& Entreprise, $\mathrm{n}^{\circ} 289$, pp. 61-79.

Geindre S. (2005). Le rôle de l'acteur tiers dans la construction d'un réseau stratégique. Revue Française de Gestion, n ${ }^{\circ}$ 154, pp. 75-91.

Glaser B., Strauss A. (1967). The discovery of grounded theory. Strategies for qualitative research. Chicago (IL), Aldine.

Granata J. (2010). Déterminants, modalités et performance des stratégies collectives en PME : le cas du syndicat des producteurs de vin du Pic Saint-Loup. Thèse de doctorat en Sciences de Gestion, Université Montpellier I, novembre.

Granata J., Le Roy F. (2014). Le management de la coopétition en PME : le cas des vignerons du Pic Saint-Loup. Finance Contrôle Stratégie, vol. 17, n 2, pp. 1-16.

Greenwood R., Suddaby R., Hinings C. (2002). Theorizing change: the role of professional associations in the transformation of institutionalized fields. Academy of Management Journal, vol. 45, n 1 , pp. 58-80.

Groené D. (1983). Le liège en Corse. Revue Forestière Française, vol. 35, n ${ }^{\circ}$, pp. 299-307.

Guillemette F. (2006). L'approche de la grounded theory; pour innover? Recherches qualitatives, vol. 26, $\mathrm{n}^{\circ} 1$, pp. 32-50.

Gundolf K., Jaouen A., Loup S. (2006). Institutions locales et TPE dans le cas du tourisme. Revue Française de Gestion, $\mathrm{n}^{\circ} 167$, p. 141-157.

Hurwicz L. (1987). Inventing new institutions: the design perspective. American Journal 
of Agricultural Economics, vol. 69, $\mathrm{n}^{\circ} 2$, pp. 395-402.

Inventaire Forestier National (2009). La forêt française : les résultats issus des campagnes d'inventaire 2005-2009, région Corse. Paris, Institut National de l'Information Géographique et Forestière.

Jarillo J.-C. (1988). On strategic networks. Strategic Management Journal, vol. 9, $\mathrm{n}^{\circ} 1$, pp. 31-41.

Lambert-Derkimba A., Verrier É., Casabianca F. (2011). Tensions entre ressources génétiques locales et ancrage territorial des produits : la race porcine corse dans un projet AOP. Économie rurale, $\mathrm{n}^{\circ} 322$, pp. 39-49.

Lecocq X. (2003). Comportement d'acteurs et dynamique d'un réseau inter-organisationnel : le phénomène des écarts relationnels. Thèse de doctorat en Sciences de Gestion, Université des Sciences et Technologies de Lille I, novembre.

Le Roy F. (2003). Rivaliser et coopérer avec ses concurrents : le cas des stratégies collectives agglomérées. Revue Française de Gestion, $\mathrm{n}^{\circ} 143$, pp. 145-157.

Le Roy F. (2008). The rise and fall of collective strategies: a case study. International Journal of Entrepreneurship \& Small Business, vol. 5, n 2, pp. 127-142.

Loubaresse É. (2008). Influence des caractéristiques des pilotes de réseaux locaux d'organisations sur leurs rôles. Management International, vol. 13, $\mathrm{n}^{\circ}$ 1, pp. 85-96.

Loubaresse É., Pestre F. (2014). Les facteurs de réussite d'une stratégie collective : le cas d'Interloire. In S. Charters, V. Michaux (dir.), Stratégies des territoires vitivinicoles : clusters, gouvernance et marque territoriale. Caen, Éditions Management \& Société, pp. 209-222.

Loup S. (2003). Les petites entreprises des métiers d'art. Revue Française de Gestion, n 144, pp. 195-209.

Ménard C. (2003). L'approche néo-institutionnelle : des concepts, une méthode, des résultats. Cahiers d'Économie Politique, $\mathrm{n}^{\circ} 44$, pp. 1-16.

Meyer J., Rowan B. (1977). Institutional organizations: formal structure as myth and ceremony. American Journal of Sociology, vol. 83, n 2, pp. 340-363.

Miles R., Snow C. (1986). Organizations: new concepts for new firms. California Management Review, vol. 28, n 3, pp. 62-73.

Mizuno Y. (2014). Collective strategy for implementing innovation in SMEs. Annals of Business Administrative Science, vol. 13, n 3, pp. 153-168.

Moore J. (1993). Predators and prey: a new ecology of competition. Harvard Business Review, vol. 71, $\mathrm{n}^{\circ} 3$, pp. 75-86.

Moisander J. (2007). Motivational complexity of green consumerism. International Journal of Consumer Study, vol. 31, $\mathrm{n}^{\circ} 4$, pp. 404-409.

Normandin D. (1980). L'économie du liège en France. Revue Forestière Française, vol. 32, $\mathrm{n}^{\circ} 1$, pp. 79-90.

North D. (1990). Institutions, institutional change, and economic performance. Cambridge, Cambridge University Press.

Orléan A. (1994). Sur le rôle respectif de la confiance et de l'intérêt dans la construction de l'ordre marchand. Revue du MAUSS, $\mathrm{n}^{\circ} 4$, pp. 17-36.

Pérez R. (1983). Introduction méthodologique sur l'articulation filières-stratégies. In ADEFI (dir.), L'analyse de filière. Paris, Economica, pp. 69-74.

Piazzetta R. (2005). État des lieux de la filière liège française. Vives, Institut Méditerranéen du Liège.

Piazzetta R. (2009). Dossier : le liège. Forêts de France, $n^{\circ}$ 521, pp. 20-29.

Powell W., DiMaggio P. (dir.) (2012). The new institutionalism in organizational analysis. Chicago (IL), University of Chicago Press.

Ring P., Van de Ven A. (1992). Structuring cooperative relationships between organizations. Strategic Management Journal, vol. 13, n 7, pp. 483-498.

Ring P., Van de Ven A. (1994). Developmental processes of cooperative interorganizational relationships. Academy of Management Review, vol. 19, n 1, pp. 90-118.

Romelaer P. (2005). L'entretien de recherche. In P. Roussel, F. Wacheux (dir.), Management 
RECHERCHE

Laetitia DARI, Gilles PACHÉ

des ressources humaines. Bruxelles, De Boeck, pp. 101-137.

Roy P. (2004). Les stratégies collectives : moyen de générer ou de s'affranchir de l'interdépendance ? Le cas des salles de cinéma. Actes de la XIX ${ }^{e}$ Conférence Internationale de l'AIMS. Le Havre, pp. 1-19 (CD-rom).

Rubin H., Rubin I. (1995). Qualitative interviewing. The art of hearing data. Thousand Oaks (CA), Sage.

Sabate M. (2000). Le matériau liège : perspectives marketing et stratégiques. Actes $d u$ Colloque Vivexpo de l'Institut Méditerranéen du Liège. Vives, pp. 1-6 (CD-rom).

Scott W., Meyer J. (1983). The organization of societal sectors. In W. Scott (dir.), Organizational environments: ritual and rationality. Thousand Oaks (CA), Sage, pp. 129-153.

Sekkat K. (1996). Les relations verticales inter-entreprises : objectifs et instruments. Bruxelles, Éditions de l'Université de Bruxelles.

Snow C., Miles R., Coleman H. (1992). Managing $21^{\text {st }}$ century networks organizations. Organizational Dynamics, vol. 20, $\mathrm{n}^{\circ} 3$, pp. 5-19.

Soulié D. (1980). Filière de production et intégration verticale. Annales des Mines, $\mathrm{n}^{\circ} 1$, pp. 21-28.

Strauss A., Corbin J. (1990). Basics of qualitative research. Thousand Oaks (CA), Sage.

Suchman M. (1995). Managing legitimacy: strategic and institutional approach. Academy of Management Review, vol. 20, $\mathrm{n}^{\circ} 3$, pp. 571-610.
Théret B. (2000). Nouvelle économie institutionnelle, économie des conventions et théorie de la régulation : vers une synthèse institutionnaliste ? La Lettre de la Régulation, $\mathrm{n}^{\circ} 35$, pp. 1-5.

Tixier D., Mathe H., Colin J. (1996). La logistique d'entreprise : vers un management plus compétitif. Paris, Dunod.

Walliser B. (1989). Théorie des jeux et genèse des institutions. Recherches économiques de Louvain, vol. 55, n 4, pp. 339-364.

Williamson O. (1985). The economic institutions of capitalism: firms, markets, relational contracting. New York (NY), The Free Press.

Xhauflair V., Pichault F. (2012). Du tiers à la tercéisation : modalités d'une fonction essentielle pour l'émergence d'une régulation à l'échelon inter-organisationnel. Négociations, $\mathrm{n}^{\circ} 18$, pp. 43-59.

Yami S. (2003). Petite entreprise et stratégie collective de filière. Revue Française de Gestion, $\mathrm{n}^{\circ}$ 144, pp. 165-179.

Yami S., Le Roy F. (dir.) (2006). Stratégies collectives : rivaliser et coopérer avec ses concurrents. Caen, Éditions Management \& Société.

Yessad S. (2000). Le chêne-liège et le liège dans les pays de la Méditerranée occidentale, Louvain-la-Neuve, ASBL Forêt Wallone.

Yin R. (2013). Case study research: design and methods. Thousand Oaks (CA), Sage, $5^{\mathrm{e}}$ éd.

Zaoual A.-R. (2014). Pour une contribution du management stratégique à l'écologie industrielle. Développement Durable \& Territoires, vol. 5, $\mathrm{n}^{\circ}$ 1, pp. 1-16. 